\title{
Legal and Ethical Aspects of Wireless Health
}

\author{
Atalla K, Chaudhary A, Eshaghian-Wilner MM*, Gupta A, Mehta R, Nayak A, Ravicz K, Shiroma B and Trivedi P \\ Department of Electrical Engineering-Systems, University of Southern California, USA
}

\begin{abstract}
Nanomedicine is a revolutionary field with the potential to vastly improve healthcare worldwide. Despite these benefits, the revolutionary rather than evolutionary nature of these new medicines raises unforeseen problems relating to ethics, privacy, intellectual property, and regulatory law. Ethical issues include the risks posed to users of a drug during the research and development stage, unequal distribution of new medicines, and the possibility for genetic self-enhancement. Privacy issues involve laws that do not adequately protect people's privacy and the possibility for genetic discrimination based on increasingly accessible genetic information. Intellectual property issues are primarily concerned with the balance between protecting nanomedicine-related intellectual property rights and the desire to encourage future innovation. Nanomedicine is particularly difficult to protect from an intellectual property perspective due to bureaucratic distinctions, difficult analysis against prior art, and the complexity of the technology itself. However, the industry as well as the courts may not be enough to properly regulate this nascent field. Given the remarkable potential of nanomedicine to improve global healthcare as well as the great risks that come with new developments in the field, it is important for researchers, industry, legislatures, and the public to work together to ensure the impending medical revolution keeps people safe and changes our lives for the better.
\end{abstract}

Keywords: Ethics; Legal; Wireless; Health; Privacy

\section{Content}

While innovation in the field of nanomedicine will undoubtedly give rise to many unforeseen risks - especially in the realms of ethics and privacy - these risks do not outweigh the overall benefits of such innovation. As technology continues to evolve, many people are becoming increasingly afraid that their rights are not being adequately protected. The success of recent hacking scandals has served only to make the public less confident in the ability of many institutions to safeguard private information. Although driving the nanomedical revolution forward will create a number of new challenges to overcome, the benefits to society that this revolution promises make it the key to a brighter future.

Ethical issues arising out of the advancement of nanomedicine are the most common arguments against continuing research in the field. Ethical issues can be broken down into three subgroups: issues in the R\&D stage, issues in the distribution stage, and issues in the final application stage. In the R\&D stage, given that analysis of the untested medical product is fundamentally incomplete, researchers are unaware of all the effects it may have on trial patients. Therefore, researchers and doctors are unable to accurately inform these patients about the risks they could face and are partly responsible for adverse consequences caused by the product. In the distribution stage, existing income disparities in society could result in unequal access to the benefits of nanomedicine. Under such a system, access to certain medication would be determined by which members of society can most afford it rather than members who most need it. Income disparities also pose possible ethical issues in the final application stage. Because of the remarkable potential nanomedicines have to alter the human body, there is the possibility that people could abuse nanomedicine for self-enhancement. This could theoretically lead to a world in which the wealthiest members of society will have the greatest physical and mental abilities simply because they alone have access to certain nanomedicine.

As with ethical issues, privacy issues related to nanomedicine are often used as arguments against advancement of the field. Privacy issues can be broken down into three subgroups: privacy law, enabled genetic discrimination, and vulnerabilities in e-healthcare systems.
The difficulty in writing legislation to protect privacy runs alongside the difficulty - earlier described - in adequately informing patients about the risks associated with new drugs. That is, because so little is currently known about nanomedicine and its potential, lawmakers are unable to write laws that provide sufficient protection to the public. Because nanomedicine promises to allow analysis of genetic material from within the body and the eventual development of affordable, personalized medicine, it at the same time threatens to make this genetic material and other medical data available to an unprecedented extent. The storage of this information in e-healthcare systems is problematic given the vulnerability of such systems and the extremely private nature of the data in question. These databases can be breached and infiltrated, giving hackers access to highly personal information, such as mental health history or treatment for sexually transmitted diseases. The increased accessibility of this medical information would also inevitably open up the possibility for instances of genetic discrimination.

While all of these risks are certainly founded and should inspire caution, the benefits of nanomedicine remain expansive and groundbreaking. Cancer has long been the most feared disease, and recovery from it has always been uncertain. Although we have certainly progressed from the days when no effective treatments existed, the current methods of battling cancer can often be harmful, often involving terrible side effects. However, the use of nanotechnology in battling cancer could be a game changer.

At this moment, modern cancer treatment is anything but ideal. Current treatments such as chemotherapy, radiation, surgery and

*Corresponding author: Eshaghian-Wilner MM, Department of Electrical Engineering-Systems, University of Southern California, USA, Tel: (213) 740-6257; E-mail: eshaghia@usc.edu

Received March 23, 2016; Accepted March 25, 2016; Published April 01, 2016

Citation: Atalla K, Chaudhary A, Eshaghian-Wilner MM, Gupta A, Mehta R, et al. (2016) Legal and Ethical Aspects of Wireless Health. J Nanomed Nanotechnol 7 : 367. doi:10.4172/2157-7439.1000367

Copyright: ( 2016 Atalla K, et al. This is an open-access article distributed under the terms of the Creative Commons Attribution License, which permits unrestricted use, distribution, and reproduction in any medium, provided the original author and source are credited. 
immunotherapy indiscriminately attack all the cells in a given area. This would destroy malignant tissue, but also damage benign tissue. Furthermore, highly toxic drug concentrations can harm or kill the patient. This is why chemotherapy treatment is not determined by the dosage needed to kill all the cancerous cells but rather by the dosage that the patient can withstand.

When it comes to battling cancer, early detection has always been the easiest way to ensure full recovery and to avoid some of the more harmful treatments. Nanotechnologies like nanowires, carbon nanotubes, nanoscale cantilevers, various nanoparticles and nanorobots could allow us to successfully detect cancerous cells. Recent studies using nanorobotics to detect diseased cells and deliver drugs "promise results that are impossible at the macro scale" $[1,2]$. For example, inorganic nanorobots could have the potential to locate the exact position of a cell using the intensified magnetic property from the force of attraction inside the human body. In other words, they can isolate a target cell from a normal cell and at the same time allow for targeted drug delivery via nanorobot. Additional information regarding current and future work in the field of nanomedicine can be found in our recent publications [3-18].

Nanotechnology has the potential not only to target sick cells but also to repair damaged tissue through the use of suitable nanomaterialbased scaffolds and growth factors. If nanomedicine has the potential to make such progress in cancer, one can only imagine its uses in battling other diseases.

Given the risks involved, advancing nanomedical technology may seem intimidating and inadvisable. However, no progress would ever be made if only risks were considered. Throughout history, humanity has made great progress by acting boldly in the face of uncertainty. We must follow these historical examples to develop and refine a cure in the battle against cancer.

When Edward Jenner first experimented with the smallpox vaccine, many thought he was insane. People didn't understand how injecting yourself with a simpler version of the same virus - cowpox - could make you immune to smallpox. Although Jenner's logic was solid - not to mention vaccines had been tested throughout the years by several different civilizations - there was confusion about the potential harm to vaccine users due to the limited use of this treatment. Many didn't want to take the risk of harming themselves in the long run and out of fear, many people, including the top medical professionals of the time, went to great lengths to stop Jenner's research and any potential success. Despite these efforts, we no longer have smallpox today because many still believed in the progress of medical treatment.

We are at a similar crossroads with nanomedicine. While there are several risk factors involved, nanomedicine may finally help us battle and cure diseases that have long petrified and stumped us. Given the promise that nanomedicine holds, it is likely that society will be able to rise to this regulatory challenge and that nanomedicine will be able to provide society with the hopeful and healthful future that so many predict it will.

\section{References}

1. Atalla K, Chaudhary A, Eshaghian-Wilner MM, Gupta A, Mehta R (2016) Ethical/Legal Implications of Wireless Health. $13^{\text {th }}$ International Conference on Nanotek and Expo, Arizona, USA

2. Eshaghian-Wilner M (2016) Wireless Computing in Medicine: From Nano to Cloud with Ethical and Legal Implications. Wiley.

3. Ahuja N, Eshaghian-Wilner MM, Ge Z, Liu R, Patil ASN (2016) Wireless Power for Body Devices. th $^{\text {th }}$ Nano Congress for Next Generation, Manchester, UK.
4. Bairy B, Craig TS, Eshaghian-Wilner MM, Gonde K, Gupta N, et al. (2016) Towards mitigating the impact of NBTI and PBTI Degradation. $2^{\text {nd }}$ World Congress on Automation and Robotics Conference, Pennsylvania, USA.

5. Craig TS, Eshaghian-Wilner MM, Goli N, Gupta A, Reddy CSK (2016) Security in Pervasive Healthcare Systems. $8^{\text {th }}$ World Medical Nanotechnology Congress and Expo, Dallas, USA

6. Bhargava A, Cheung J, Lee W, Ravicz K, Schlesinger M, et al. (2016) Status and modeling of Nanomedicine. $6^{\text {th }}$ Global Experts Meeting \& Expo on Nanomaterials and Nanotechnology, Dubai.

7. Eshaghian-Wilner MM, Ge C, Liu R, Pati ASN (2016) Wireless Power for Implantable Devices: A Technical Review. Wireless Computing in Medicine and Its Ethical/Legal Implications, Wiley.

8. Bhargava A, Eshaghian-Wilner MM, Pati ASN (2016) An Introduction to Wireless Computing in Medicine. Wireless Computing in Medicine and Its Ethical/Legal Implications, Wiley.

9. Eshaghian-Wilner MM, Ge Z, Liu R (2016) Wireless Power for Medicine Wireless Computing in Medicine and Its Ethical/Legal Implications: From Nano to Cloud, Wiley.

10. Eshaghian-Wilner MM, Navab S, Sarkar G (2016) Cloud to Nano Wireless Computing. Wireless Computing in Medicine and Its Ethical/Legal Implications: From Nano to Cloud, Wiley.

11. Bhargava A, Cheung J, Eshaghian-Wilner MM, Lee W, Padala KSR, et al (2016) Nanotechnology in Medicine to appear in an edited volume on "Wireless Computing in Medicine and Its Ethical/Legal Implications: From Nano to Cloud.

12. Bhargava A, Cheung J, Eshaghian-Wilner MM, Lee W, Schlesinger M, et al. (2016) An Introduction to Nanomedicine, to appear in an edited volume on "Wireless Computing in Medicine and Its Ethical/Legal Implications: From Nano to Cloud.

13. Eshaghian-Wilner MM, Khizroev S, Sarkar G, Sharma U (2016) Efficient Ondemand Drug Delivery Systems Using Magneto-electric Nano Particles, to appear in an edited volume on "Wireless Computing in Medicine and Its Ethical/ Legal Implications: From Nano to Cloud.

14. Eshaghian-Wilner MM, Schlesinger M (2016) Concluding Remarks and Future Research in Wireless Computing in Medicine. Wireless Computing in Medicine and Its Ethical/Legal Implications: From Nano to Cloud Wiley.

15. Ahuja N, Eshaghian-Wilner MM, Ge Z, Liu R, Pati ASN, et al. (2016) Toward the Design of Body Devices Wireless Power, International Journal of Nanomedicine and Nanosurgery.

16. Bairy B, Craig TS, Eshaghian-Wilner MM, Gonde K, Gupta N, et al. (2016) Mitigating the impact of NBTI and PBTI Degradation. I Global Journal of Technology and Optimization.

17. Craig TS, Eshaghian-Wilner MM, Goli N, Gupta A, Reddy CSK (2016) Securing Pervasive Communications in Healthcare Systems. European Journal of Advances in Engineering and Technology.

18. Bhargava A, Janet Cheung, Mary Mehrnoosh Eshaghian-Wilner, Wan Lee Kodiak Ravicz, Mike Schlesinger, Yesha Shah, Abhishek Uppal (2016) Review of Nanomedicine. International Journal of Nano Studies and Technology. 